\title{
Qualidade de ovos de poedeiras comerciais armazenados em diferentes temperaturas e períodos de estocagem
}

\author{
Quality of eggs from commercial laying hens stored in different periods of temperature \\ and storage
}

\author{
LANA, Sandra Roseli Valerio $^{1 *}$; LANA, Geraldo Roberto Quintão ${ }^{1}$; SALVADOR, \\ Edivânia de Lima ${ }^{1}$; LANA, Ângela Maria Quintão ${ }^{2}$; CUNHA, Fabio Sales \\ Albuquerque $^{3}$; MARINHO, Andreza Lourenço ${ }^{1}$
}

${ }^{1}$ Universidade Federal de Alagoas, Departamento de Zootecnia, Maceió, Alagoas, Brasil.

${ }^{2}$ Universidade Federal de Minas Gerais, Departamento de Zootecnia, Belo Horizonte, Minas Gerais, Brasil.

${ }^{3}$ Universidade Estadual de Alagoas, Departamento de Zootecnia, Santana do Ipanema, Alagoas,Brasil

*Endereço para correspondência: svlana@bol.com.br

\section{RESUMO}

Objetivou-se avaliar a qualidade dos ovos de poedeiras comerciais armazenados em diferentes temperaturas e períodos de armazenamento. Foram coletados, logo após a postura, 360 ovos de galinhas poedeiras da linhagem Dekalb White. Todos os ovos foram identificados, pesados e distribuídos aleatoriamente às bandejas, sendo 150 armazenados em temperatura ambiente (26,5 $\left.\pm \quad 0,7^{\circ} \mathrm{C}\right), \quad 150$ ovos acondicionados em refrigeração $\left(7,3 \pm 0,5^{\circ} \mathrm{C}\right)$ e 60 ovos separados para realizar a avaliação no primeiro dia. $\mathrm{O}$ delineamento utilizado foi inteiramente casualizado em esquema fatorial $2 \times 6$, sendo duas temperaturas de armazenamento e seis períodos de avaliação da estocagem (zero, seis, $12,18,24$, e 30 dias), com 30 repetições. As variáveis analisadas foram: pesos dos ovos, pesos absolutos e relativos de albúmen, gema e casca, altura do albúmen, gravidade específica, Unidade Haugh, diâmetro de albúmen, índice de albúmen e de gema. Os valores de pesos absolutos e relativos de ovos, gema e albúmen, altura de albúmen, gravidade específica, Unidade Haugh, diâmetro e índices de albúmen e de gema foram influenciados $(\mathrm{P}<0,05)$ pela temperatura e período de armazenamento. Os pesos absolutos de cascas não $(\mathrm{P}>0,05)$ foram influenciados pelas diferentes temperaturas, entretanto, as porcentagens de casca foram influenciadas $(\mathrm{P}<0,05)$ linearmente pelo tempo de armazenamento dos ovos. Concluiu-se que os ovos armazenados até seis dias após a postura se mantêm em padrão de alta qualidade em temperatura ambiente $\left(26,5^{\circ} \mathrm{C}\right)$, e quando armazenados em refrigeração $\left(7,3^{\circ} \mathrm{C}\right)$ mantêm padrão de excelente qualidade até os 30 dias.

Palavras-chave: gravidade específica, índice de albúmen e gema, unidade Haugh

\section{SUMMARY}

The study aimed at assessing the quality of commercial laying hen eggs stored under different temperatures and for different storage periods. 360 eggs were collected soon after laying by DeKalb White laying hens. All eggs were identified, weighed, and randomly distributed in trays, being 150 stored at room temperature $\left(26.5 \pm 0.7^{\circ} \mathrm{C}\right), 150$ other placed in trays under refrigeration $\left(7.3 \pm 0.5^{\circ} \mathrm{C}\right)$, and the remaining 60 eggs were set apart so the assessment could be performed on the first day. The trial design was completely randomized $2 \times 11$, scheme factorial, with two storage temperatures and six storage periods (zero, six, $12,18,24$ and 30 days), with 30 repetitions. The assessed variables were egg weights, absolute and relative weights of albumen, yolk and shell, albumen height, specific gravity, Haugh unit, albumen diameter, albumen index and yolk. The values of absolute and relative weights of egg, albumen and yolk, albumen height, specific gravity, Haugh Unit, diameter and indices of albumen and yolk were significantly influenced 
$(\mathrm{P}<0.05)$ by the storage temperature and period. The absolute weight does not of shell $(\mathrm{P}>0.05)$ were influenced by different temperatures, however, the percentages of shell were influenced $(\mathrm{P}<0.05)$ linearly by storage time of eggs. It can be concluded that the eggs maintain high quality when stored for up to six days after laying, at room temperature, and maintain excellent quality when stored under refrigeration for up to 30 days.

Keywords: albumen and yolk index, Haugh unit, specific gravity

\section{INTRODUCÃO}

Como todos os produtos de origem animal, o ovo é perecível, e começa a perder sua qualidade interna após a postura, caso não sejam tomadas medidas adequadas para sua conservação. Assim, a perda de qualidade é um fenômeno inevitável que acorre de forma contínua ao longo do tempo e pode ser agravado pela temperatura, umidade relativa e estado nutricional da poedeira.

No Brasil, por não ser obrigatória a refrigeração, os ovos são acondicionados, desde o momento da postura até a distribuição final, em temperatura ambiente e refrigerados na casa do consumidor. Embora a legislação brasileira (BRASIL, 1997) determine condições mínimas internas, como câmaras de ar de quatro a $10 \mathrm{~mm}$ de altura; gema translúcida e consistente; clara transparente, consistente, sem mancha, na prática, apenas o peso e características aparentes de casca (sujeiras, trincas e cascas defeituosas) têm sido considerados. A utilização de unidade Haugh (UH), que é a altura do albúmen corrigida para o peso do ovo, como avaliação da qualidade interna, é universal devido à sua fácil aplicação e à alta correlação com a aparência do ovo ao ser quebrado, e é definida como aferidor da qualidade interna do ovo e tem sido utilizada pela indústria desde sua introdução em 1937.

O programa de controle da qualidade preconizado pelo USDA define as condições ideais, desde a produção do ovo até seu consumo pela população, assim, ovos considerados de qualidade excelente, alta e baixa devem apresentar, respectivamente, valores de $\mathrm{UH}$ acima de 72 ; entre 60 e 72; e menores que 60 (USDA, 2000).

A velocidade das alterações no albúmen e gema está associada à temperatura e ao movimento de dióxido de carbono do albúmen através da casca, em consequência de um gradiente negativo de concentração (KEENER et al., 2001). Estudos realizados por Figueiredo et al. (2011), Pissinati et al. (2014) e Giampietro-Ganeco et al. (2015) para avaliar os efeitos da temperatura e do tempo de armazenamento sobre a qualidade dos ovos, demonstraram que maior tempo de estocagem e $\mathrm{o}$ acondicionamento à temperatura ambiente, promoveram alteração na qualidade, como queda nos valores de $\mathrm{U}$ $\mathrm{H}$, redução da altura de albúmen $\mathrm{e}$ elevação de $\mathrm{pH}$ do albúmen, evidenciando a importância da refrigeração para preservar sua qualidade, e garantir ao consumidor um produto saudável.

Dentro deste contexto, objetivou-se avaliar a qualidade interna de ovos de poedeiras comerciais, para consumo humano, armazenados em diferentes temperaturas e períodos de armazenamento.

\section{MATERIAL E MÉTODOS}

O experimento foi conduzido no Laboratório de Nutrição Animal da Unidade Acadêmica Centro de Ciências 
Agrárias da Universidade Federal de Alagoas - UFAL.

Foram coletados aleatoriamente 360 ovos, provenientes da primeira coleta do dia, de galinhas poedeiras da linhagem Dekalb White, com 26 semanas de idade, procedentes da Granja Carnaúba, localizada em União dos Palmares, AL. Todos os ovos foram identificados, pesados em balança de precisão e em seguida, acondicionados em bandejas de papelão com capacidade de três dúzias cada. Os ovos foram distribuídos aleatoriamente às bandejas, sendo 150 armazenados em temperatura ambiente, 150 ovos acondicionados em refrigeração e 60 ovos para realizar a avaliação imediatamente após à postura (zero dia).

Durante todo o período experimental as temperaturas máximas e mínimas dos locais de armazenamento foram registradas diariamente as $10 \mathrm{~h} 00 \mathrm{~min}$ horas por meio de termo-higrômetro digital da marca Incoterm. A média da temperatura de refrigeração foi de 7,3 \pm $0,5^{\circ} \mathrm{C}$, e a da temperatura ambiente de $26,5 \pm 0,7^{\circ} \mathrm{C}$.

$\mathrm{O}$ delineamento utilizado foi $\mathrm{o}$ inteiramente casualizado em esquema fatorial $2 \times 6$, sendo duas condições de armazenamento (temperatura ambiente e de refrigeração) e seis períodos de avaliação da estocagem (zero, seis, 12, 18,24 e 30 dias), com 30 repetições por tratamento e o ovo considerado a repetição. As variáveis analisadas foram os pesos dos ovos, pesos absolutos e relativos de albúmen, gema e casca, altura do albúmen, gravidade específica, unidade Haugh, diâmetro de albúmen, índice de albúmen e de gema.

Ao final de cada período de armazenamento, ou seja, $0 ; 6 ; 12 ; 18 ; 24$ e 30 dias de estocagem procedeu-se a determinação dos pesos dos ovos, de albúmen, gema e casca, utilizando uma balança analítica da marca Mettler. Para os cálculos das porcentagens de casca, gema e de albúmen utilizou-se, respectivamente, as seguintes fórmulas: $\%$ casca $=$ (pesodacasca $/$ pesodoovo $) * 100$, $\%$ gema $=($ pesodagema/pesodo ovo $) * 100$, e $\%$ albúmen $=100-(\%$ gema $+\%$ casca $)$. Após a quebra dos ovos as cascas foram lavadas cuidadosamente com água, colocadas em estufa ventilada à $55^{\circ} \mathrm{C}$ por 12 horas para secagem. Em seguida as cascas foram retiradas da estufa e mantidas à temperatura ambiente por duas horas para proceder-se a pesagem das mesmas.

A gravidade específica foi determinada pelo método da flutuação salina, conforme metodologia descrita por Hamilton (1982). Em seguida, os ovos foram quebrados em uma superfície plana e lisa de vidro, onde se realizou, as medidas de altura do albúmen denso e da gema em milímetros por um micrômetro digital da marca Digimes, com resolução $0,01 \mathrm{~mm} / .0005$ " acoplado a uma base tripé. Com a medida do albúmen e do peso do ovo, foram determinados os valores de unidade Haugh pela fórmula $\mathrm{UH}=100 \mathrm{log}$ $\left(\mathrm{H}+7.57-1.7 \mathrm{~W}^{0.37}\right.$, em que $\mathrm{H}=$ altura do albúmen $(\mathrm{mm})$ e $\mathrm{W}=$ peso do ovo $(\mathrm{g})$ (ROMANOFF \& ROMANOFF, 1963; SILVERSIDES et al., 1993). Os diâmetros do albúmen denso e da gema foram obitidos por meio do paquímetro digital da marca Digimess, assim para obter o valor do índice de albúmen e gema, dividiu-se suas respectivas alturas pelos valores médios de seus diâmetros.

As análises estatísticas das variáveis estudadas foram realizadas utilizando o programa SAEG - Sistema para Análises Estatísticas, versão 9.1 e as médias foram comparadas pelo teste Student Newman Keuls a 5\% de probabilidade. 


\section{RESULTADOS E DISCUSSÃO}

Os pesos de gema e de albúmen dos ovos foram influenciados $(\mathrm{P}<0,05)$ pelas diferentes temperaturas e períodos de estocagem (Tabela 1).

Os ovos armazenados em temperatura ambiente apresentaram maiores $(\mathrm{P}<0,05)$ valores de peso de gema que os ovos mantidos em refrigeração, evidenciando que o peso da gema dos ovos é influenciado tanto pela temperatura quanto pelo tempo de armazenamento. Pode-se verificar que em valores absolutos foi constatado aumento de 8,4 e $11,2 \%$ no peso de gema dos ovos armazenados entre o $6^{\circ} \mathrm{e}$ o $30^{\circ}$ dia, em temperatura ambiente e refrigerada, respectivamente.

Tabela 1. Valores de pesos absolutos e relativos dos componentes de ovos de poedeiras comerciais armazenados em diferentes temperaturas e períodos de estocagem

\begin{tabular}{|c|c|c|c|c|c|c|c|c|c|}
\hline \multirow{2}{*}{$\begin{array}{l}\text { Componentes do } \\
\text { ovo }\end{array}$} & \multirow{2}{*}{$\begin{array}{c}\text { Temperatura } \\
\left({ }^{\circ} \mathrm{C}\right)\end{array}$} & \multicolumn{6}{|c|}{ Períodos de estocagem (dias) } & \multirow{2}{*}{ Médias } & \multirow{2}{*}{$\begin{array}{l}\text { CV } \\
(\%)\end{array}$} \\
\hline & & 0 & 06 & 12 & 18 & 24 & 30 & & \\
\hline \multirow{2}{*}{ Gema $(g)^{*}$} & 26,5 & $13,97^{\mathrm{a}}$ & $14,29^{\mathrm{a}}$ & $14,35^{\mathrm{a}}$ & $14,34^{\mathrm{a}}$ & $15,01^{\mathrm{a}}$ & $15,49^{\mathrm{a}}$ & $14,58^{\mathrm{A}}$ & \multirow{2}{*}{6,89} \\
\hline & 7,3 & $13,34^{\mathrm{a}}$ & $13,47^{\mathrm{b}}$ & $13,62^{\mathrm{b}}$ & $14,19^{\mathrm{a}}$ & $14,36^{\mathrm{b}}$ & $14,98^{\mathrm{b}}$ & $13,99^{\mathrm{B}}$ & \\
\hline \multirow{2}{*}{ Albúmen $(\mathrm{g})^{*}$} & 26,5 & $35,80^{\mathrm{a}}$ & $33,81^{\mathrm{b}}$ & $33,31^{\mathrm{b}}$ & $32,40^{\mathrm{b}}$ & $30,76^{\mathrm{b}}$ & $30,18^{b}$ & $32,71^{\mathrm{B}}$ & \multirow{2}{*}{3,95} \\
\hline & 7,3 & $35,40^{\mathrm{a}}$ & $34,91^{\mathrm{a}}$ & $34,33^{\mathrm{a}}$ & $32,89^{\mathrm{a}}$ & $32,25^{\mathrm{a}}$ & $31,37^{\mathrm{a}}$ & $33,53^{\mathrm{A}}$ & \\
\hline \multirow{2}{*}{ Casca $(g)^{\mathrm{ns}}$} & 26,5 & $5,47^{\mathrm{a}}$ & $5,71^{\mathrm{a}}$ & $5,58^{\mathrm{a}}$ & $5,74^{\mathrm{a}}$ & $5,51^{\mathrm{a}}$ & $5,50^{\mathrm{a}}$ & $5,59^{\mathrm{A}}$ & \multirow{2}{*}{6,62} \\
\hline & 7,3 & $5,46^{\mathrm{a}}$ & $5,50^{\mathrm{a}}$ & $5,63^{\mathrm{a}}$ & $5,50^{\mathrm{a}}$ & $5,58^{\mathrm{a}}$ & $5,48^{\mathrm{a}}$ & $5,53^{\mathrm{A}}$ & \\
\hline \multirow{2}{*}{ Peso do ovo $(\mathrm{g})^{*}$} & 26,5 & $55,24^{\mathrm{a}}$ & $53,81^{\mathrm{a}}$ & $53,24^{\mathrm{a}}$ & $52,48^{a}$ & $51,28^{\mathrm{a}}$ & $51,17^{\mathrm{a}}$ & $52,87^{\mathrm{A}}$ & \multirow{2}{*}{2,37} \\
\hline & 7,3 & $54,20^{\mathrm{a}}$ & $53,88^{a}$ & $53,58^{\mathrm{a}}$ & $52,58^{\mathrm{a}}$ & $52,19^{\mathrm{a}}$ & $51,83^{\mathrm{a}}$ & $53,04^{\mathrm{A}}$ & \\
\hline \multirow{2}{*}{ Gema $(\%)^{*}$} & 26,5 & $25,29^{\mathrm{a}}$ & $26,56^{\mathrm{a}}$ & $26,95^{\mathrm{a}}$ & $27,32^{\mathrm{a}}$ & $29,27^{\mathrm{a}}$ & $30,27^{\mathrm{a}}$ & $27,61^{\mathrm{A}}$ & \multirow{2}{*}{6,72} \\
\hline & 7,3 & $24,61^{\mathrm{a}}$ & $25,00^{\mathrm{b}}$ & $25,42^{b}$ & $26,99^{\mathrm{b}}$ & $27,51^{b}$ & $28,90^{\mathrm{b}}$ & $26,26^{\mathrm{B}}$ & \\
\hline \multirow{2}{*}{ Albúmen (\%)* } & 26,5 & $64,81^{\mathrm{a}}$ & $62,83^{\mathrm{b}}$ & $62,57^{b}$ & $61,74^{\mathrm{b}}$ & $59,98^{\mathrm{b}}$ & $58,98^{\mathrm{b}}$ & $61,82^{\mathrm{B}}$ & \multirow{2}{*}{2,95} \\
\hline & 7,3 & $65,31^{\mathrm{a}}$ & $64,79^{a}$ & $64,07^{\mathrm{a}}$ & $62,55^{\mathrm{a}}$ & $61,79^{\mathrm{a}}$ & $60,52^{\mathrm{a}}$ & $63,17^{\mathrm{A}}$ & \\
\hline \multirow{2}{*}{ Casca $(\%)^{*}$} & 26,5 & $9,90^{\mathrm{a}}$ & $10,61^{\mathrm{a}}$ & $10,48^{\mathrm{a}}$ & $10,94^{\mathrm{a}}$ & $10,74^{\mathrm{a}}$ & $10,75^{\mathrm{a}}$ & $10,57^{\mathrm{A}}$ & \multirow{2}{*}{6,36} \\
\hline & 7,3 & $10,07^{\mathrm{a}}$ & $10,21^{\mathrm{a}}$ & $10,51^{\mathrm{a}}$ & $10,46^{\mathrm{a}}$ & $10,69^{\mathrm{a}}$ & $10,57^{\mathrm{a}}$ & $10,42^{\mathrm{A}}$ & \\
\hline
\end{tabular}

Médias seguidas de letras diferentes, minúsculas ou maiúsculas na linha, para a mesma variável, diferem entre si, pelo teste Student Newman Keuls $(\mathrm{P}<0,05)$.

*Efeito linear $(\mathrm{P}<0,05) .{ }^{\text {ns }}$ não significativo $(\mathrm{P}>0,05)$.

Os pesos de gema dos ovos foram influenciados $(\mathrm{P}<0,05)$ linearmente à medida que aumentou o tempo de armazenamento tanto em temperatura ambiente quanto sob refrigeração, conforme as respectivas equações: $\hat{\mathrm{Y}}=$ $14,1213+0,04596 x \quad\left(\mathrm{R}^{2}=0,91\right)$ e $\hat{\mathrm{Y}}=$ $13,8973+0,03676 x \quad\left(R^{2}=0,90\right)$. Esses resultados provavelmente foram decorrentes do deslocamento de água, resultante das reações químicas do albúmen, para a gema que ocorrem mais rapidamente quando os ovos são armazenados em temperatura ambiente, aumentando consequentemente o peso da gema. Resultados semelhantes foram obtidos por Scott \& Silversides (2000), Figueiredo et al. (2011), Lopes et al. (2012).

Os ovos armazenados em refrigeração apresentaram maiores $(\mathrm{P}<0,05)$ pesos médios de albúmen que os ovos mantidos em condições de temperatura ambiente. A partir do sexto dia de 
estocagem foi constatado o efeito da temperatura sobre os pesos de albúmen dos ovos, sendo esse efeito evidenciado para os ovos armazenados em temperatura ambiente. Essa redução de peso do albúmen ao longo do período de estocagem acarretou em queda no peso desses ovos. Estes resultados estão de acordo com os obtidos por Scott \& Silversides (2000), Silversides \& Sott (2001), Figueiredo et al. (2011) e Lopes et al. (2012).

Os pesos de albúmen dos ovos reduziram $(\mathrm{P}<0,05) \quad$ linearmente à medida que aumentou o tempo de estocagem dos ovos submetidos a 26,5 e $7,3^{\circ} \mathrm{C}$, segundo as respectivas equações: $\hat{\mathrm{Y}}=35,4140-0,23335 x\left(\mathrm{R}^{2}=0,84\right)$ e $\hat{\mathrm{Y}}=$ $35,8385-0,14795 x\left(\mathrm{R}^{2}=0,99\right)$.

Essa rápida queda de qualidade interna pode ter ocorrido em virtude de que com a elevada temperatura de conservação e o tempo prolongado de armazenamento, as enzimas presentes no albúmen hidrolisaram as cadeias de aminoácidos, destruindo a estrutura protéica e liberando água ligada a grandes moléculas de proteínas, o que possivelmente tenha provocado a fluidificação do albúmen, perda de viscosidade do albúmen mais denso, alcalinização e alteração do sabor do ovo, corroborando com os resultados verificados por Scott \& Silversides, (2000); Oliveira et al. (2009); Estrada et al. (2010).

Os pesos da casca dos ovos não foram influenciados $(\mathrm{P}>0,05)$ pelas diferentes temperaturas e pelo período de armazenamento. Os ovos armazenados durante 30 dias, independente da temperatura de conservação, não apresentaram diferenças $(\mathrm{P}>0,05)$ em relação ao peso de casca. Estudos realizados por Oliveira et al. (2009), Figueiredo et al. (2011) e Lopes et al. (2012) sobre a qualidade de ovos de poedeiras comerciais submetidos a diferentes condições de armazenamento também demonstraram que não foram observadas diferenças significativas no peso de cascas.

Não foram observadas diferenças $(\mathrm{P}>0,05)$ no peso dos ovos em relação à temperatura de estocagem. No entanto, houve efeito $(\mathrm{P}<0,05)$ significativo do período de armazenamento sobre os pesos dos ovos. Os pesos dos ovos reduziram $(\mathrm{P}<0,05)$ de forma linear com o prolongamento do tempo de armazenamento dos ovos tanto em temperatura ambiente quanto sob refrigeração, conforme as equações: $\hat{\mathrm{Y}}=54,9856-0,13948 x\left(\mathrm{R}^{2}=0,96\right)$ e $\hat{\mathrm{Y}}=$ $54,3703-0,08746 x \quad\left(\mathrm{R}^{2}=0,97\right)$, respectivamente. Esse resultado provavelmente foi devido à transferência de umidade do albúmen para o meio externo através da casca, ocasionado pelo tempo de armazenamento dos ovos. Resultados semelhantes foram observados nas pesquisas realizadas por Silversides \& Scoot (2001), Jones \& Musgrove (2005), Samli et al. (2005), Barbosa et al. (2008), Garcia et al. (2010), Figueiredo et al. (2011) e Lopes et al. (2012).

Houve efeito $(\mathrm{P}<0,05)$ significativo para as porcentagens de gema e de albúmen dos ovos armazenados nas diferentes condições de temperatura e período de estocagem. Independente do período de estocagem as porcentagens de gema dos ovos armazenados a $26,5^{\circ} \mathrm{C}$ apresentaram maiores valores $(\mathrm{P}<0,05)$ que aqueles mantidos a $7,3^{\circ} \mathrm{C}$. $\mathrm{O}$ efeito da temperatura sobre as porcentagens de gema foi constatado somente a partir do sexto dia de armazenamento dos ovos estocados em temperatura ambiente e sob refrigeração, sendo esse efeito mais acentuado para os ovos mantidos a $26,5^{\circ} \mathrm{C}$.

As porcentagens de gema dos ovos foram influenciadas $(\mathrm{P}<0,05)$ linearmente à 
medida que se prolongou o tempo de armazenamento dos ovos submetidos tanto em temperatura ambiente quanto na refrigeração, conforme as respectivas equações: $\quad \hat{Y}=26,3174+0,1175 x$ $\left(\mathrm{R}^{2}=0,97\right)$ e $\hat{\mathrm{Y}}=25,7536+0,1075 x$ $\left(\mathrm{R}^{2}=0,93\right)$. $\mathrm{O}$ aumento na porcentagem de gema foi resultado da migração de água do albúmen para a gema. Este inicia-se logo após a postura, pois, no momento da postura, existe um gradiente de pressão osmótica entre o albúmen e a gema, que se acentua de forma progressiva, à medida que a água passa da clara para a gema, e esse aumento é acelerado em temperaturas mais elevadas. Estes resultados corroboram com os dados encontrados por Scott \& Silversides (2000), Barbosa et al. (2008), Santos et al. (2009) e Garcia et al. (2010).

Independente do período de armazenamento as porcentagens de albúmen dos ovos mantidos em condições de refrigeração apresentaram maiores $(\mathrm{P}<0,05)$ valores médios comparados com os ovos mantidos a temperatura ambiente. A partir do $6^{\circ}$ dia de armazenamento observou-se o efeito da temperatura sobre a porcentagem de albúmen para os ovos armazenados em temperatura ambiente e sob refrigeração, apesar desse efeito ter sido mais acentuado a $26,5^{\circ} \mathrm{C}$, demonstrando $\mathrm{o}$ efeito benéfico da refrigeração para manutenção da qualidade interna dos ovos durante a estocagem.

As porcentagens de albúmen dos ovos reduziram $(\mathrm{P}<0,05) \quad$ linearmente, em função do período de armazenamento dos ovos submetidos em temperatura ambiente e sob refrigeração, segundo as equações: $\hat{Y}=64,0131-0,17948 x$ $\left(\mathrm{R}^{2}=0,94\right)$ e $\hat{\mathrm{Y}}=63,899-0,1444 x$ $\left(\mathrm{R}^{2}=0,95\right)$, respectivamente. Conforme mencionado anteriormente, tal fato devese à transferência de água do albúmen para a gema, através da membrana vitelínica, ocasionando redução na proporção do albúmen com o tempo de armazenamento dos ovos. Esses dados são coerentes com os verificados por Silversides \& Scott (2001), Santos et al. (2009), Ramos et al. (2010) e Freitas et al. (2011).

Não houve diferenças $(\mathrm{P}>0,05)$ significativas sobre os valores médios de porcentagens de casca dos ovos armazenados nas diferentes temperaturas. Entretanto, as porcentagens de casca dos ovos foram influenciadas $(\mathrm{P}<0,05)$ linearmente à medida que se prolongou $\mathrm{o}$ tempo de armazenamento dos ovos submetidos tanto em temperatura ambiente quanto em refrigeração, conforme as respectivas equações: $\hat{\mathrm{Y}}=$ $9,9209+0,01998 x \quad\left(\mathrm{R}^{2}=0,91\right) \quad$ e $\quad \hat{\mathrm{Y}}=$ $10,1374+0,01863 x \quad\left(\mathrm{R}^{2}=0,90\right) . \quad$ Esses dados corroboram aos encontrados por Scott \& Silversides (2000), Silversides \& Scott (2001) e Ramos et al. (2010) que não verificaram efeito significativo na porcentagem de casca para ovos que foram armazenados em diferentes temperaturas. Por outro lado, Santos et al. (2009), Garcia et al. (2010) e Figueiredo et al. (2011) constataram maior proporção de casca de ovos a medida que aumentou o período de armazenamento. De acordo com os autores o resultado foi reflexo da menor perda de peso dos ovos mantidos sob refrigeração, fazendo com que o peso da casca não aumente sua proporção no peso total do ovo.

Os valores de altura de albúmen, gravidade específica e unidade Haugh dos ovos de poedeiras foram influenciados $(\mathrm{P}<0,05)$ pelas diferentes temperaturas de conservação e períodos de armazenamento (Tabela 2).

Observou-se que, independente do período de estocagem, os ovos armazenados em condições de refrigeração apresentaram maiores $(\mathrm{P}<0,05)$ valores de altura de albúmen que os ovos estocados em temperatura ambiente. Constatou-se a partir do $6^{\circ}$ 
dia de armazenamento maior queda dos valores de altura de albúmen dos ovos mantidos a $26,5^{\circ} \mathrm{C}$, quando comparados com os valores daqueles mantido em refrigeração. Dessa forma, as condições de armazenamento, como a refrigeração, podem ser importantes para a avaliação da qualidade do ovo, mantendo assim uma maior vida de prateleira do produto.

Tabela 2. Valores de altura albúmen, gravidade específica e unidade Haugh de ovos de poedeiras comerciais armazenados em diferentes temperatura e períodos de estocagem

\begin{tabular}{|c|c|c|c|c|c|c|}
\hline \multirow{2}{*}{$\begin{array}{l}\text { Período de } \\
\text { armazenam } \\
\text { ento (dias)* }\end{array}$} & \multicolumn{2}{|c|}{ Altura de Albúmen (mm) } & \multicolumn{2}{|c|}{$\begin{array}{l}\text { Gravidade } \\
\text { Específica }\end{array}$} & \multicolumn{2}{|c|}{ Unidade Haugh } \\
\hline & $26,5^{\circ} \mathrm{C}$ & $7,3^{\circ} \mathrm{C}$ & $26,5^{\circ} \mathrm{C}$ & $7,3^{\circ} \mathrm{C}$ & $26,5^{\circ} \mathrm{C}$ & $7,3^{\circ} \mathrm{C}$ \\
\hline 0 & $8,82^{\mathrm{a}}$ & $8,82^{\mathrm{a}}$ & $1,092^{\mathrm{a}}$ & $1,093^{\mathrm{a}}$ & $94,62^{\mathrm{a}}$ & $94,36^{\mathrm{a}}$ \\
\hline 6 & $4,52^{\mathrm{b}}$ & $7,06^{\mathrm{a}}$ & $1,083^{\mathrm{a}}$ & $1,084^{\mathrm{a}}$ & $66,11^{\mathrm{b}}$ & $85,42^{\mathrm{a}}$ \\
\hline 12 & $2,71^{\mathrm{b}}$ & $6,41^{\mathrm{a}}$ & $1,068^{\mathrm{b}}$ & $1,078^{\mathrm{a}}$ & $45,10^{\mathrm{b}}$ & $81,40^{\mathrm{a}}$ \\
\hline 18 & $2,80^{\mathrm{b}}$ & $6,27^{\mathrm{a}}$ & $1,074^{\mathrm{a}}$ & $1,074^{\mathrm{a}}$ & $47,06^{\mathrm{b}}$ & $80,31^{\mathrm{a}}$ \\
\hline 24 & $1,95^{\mathrm{b}}$ & $5,94^{\mathrm{a}}$ & $1,064^{\mathrm{b}}$ & $1,068^{\mathrm{a}}$ & $34,70^{\mathrm{b}}$ & $78,68^{\mathrm{a}}$ \\
\hline 30 & $1,78^{\mathrm{b}}$ & $5,97^{\mathrm{a}}$ & $1,054^{\mathrm{b}}$ & $1,060^{\mathrm{a}}$ & $30,82^{\mathrm{b}}$ & $79,19^{\mathrm{a}}$ \\
\hline Médias & $3,76^{\mathrm{B}}$ & $6,75^{\mathrm{A}}$ & $1,073^{\mathrm{B}}$ & $1,076^{\mathrm{A}}$ & $53,07^{\mathrm{B}}$ & $83,23^{\mathrm{A}}$ \\
\hline CV (\%) & \multicolumn{2}{|c|}{14,63} & \multicolumn{2}{|c|}{0,53} & \multicolumn{2}{|c|}{10,20} \\
\hline
\end{tabular}

Médias seguidas de letras diferentes na coluna, para a mesma variável, diferem entre si, pelo teste Student Newman Keuls $(\mathrm{P}<0,05)$.

* Efeito linear $(\mathrm{P}<0,05)$.

A altura de albúmen dos ovos reduziu $(\mathrm{P}<0,05)$ linearmente à medida que se prolongou o tempo de estocagem em temperatura ambiente e sob refrigeração, segundo as respectivas equações:

$$
\begin{aligned}
& \hat{\mathrm{Y}}=2,2052+6,7772 \frac{1}{x} \quad\left(\mathrm{R}^{2}=0,94\right) \\
& \hat{\mathrm{Y}}=6,1052+2,7856 \frac{1}{x} \quad\left(\mathrm{R}^{2}=0,93\right)
\end{aligned}
$$

As inúmeras reações químicas que ocorrem no albúmen, acarretam a fluidificação e elevação do $\mathrm{pH}$, permitindo a água migrar para a gema, reduzindo o peso e altura de albúmen. Esses resultados foram coerentes aos obtidos por Scott \& Silversides (2000). Houve efeito significativo $(\mathrm{P}<0,05)$ da temperatura e do período de armazenamento sobre a gravidade específica dos ovos. Observou-se que os ovos armazenados em condições de temperatura ambiente apresentaram menores valores de gravidade específica que os ovos armazenados sob refrigeração. A partir do $12^{\circ}$ dia de armazenamento foi constatado redução acentuada dos valores de gravidade específica, em ambas condições de conservação, sendo que os ovos armazenados em temperatura ambiente apresentaram menores índices que os mantidos sob refrigeração.

Os valores de gravidade específica foram reduzidos linearmente $(\mathrm{P}<0,05)$ à medida que se prolongou o tempo de armazenamento dos ovos em condições de temperatura ambiente e de refrigeração, segundo as equações: $\hat{Y}=$ $1,0977-0,00137 x\left(\mathrm{R}^{2}=0,99\right)$ e $\hat{\mathrm{Y}}=1,0971-$ $0,00117 x \quad\left(\mathrm{R}^{2}=0,93\right), \quad$ respectivamente. Resultados semelhantes foram obtidos por Samli et al. (2005), Barbosa et al. (2008) e Santos et al. (2009) e Freitas et al (2011) ao demonstrarem que a perda de 
água que ocorre no ovo depois da postura em consequência da evaporação provoca um aumento progressivo da câmara de ar e, consequentemente, a diminuição da gravidade específica do ovo. Além disso, essa redução pode estar relacionada à perda de peso dos ovos durante o armazenamento.

Os valores de Unidade Haugh foram influenciados $(\mathrm{P}<0,05)$ pelas diferentes temperaturas e períodos de armazenamento. Os ovos armazenados em condições de refrigeração apresentaram um maior valor médio de Unidade Haugh, demonstrando o benefício da utilização da refrigeração na manutenção da qualidade interna dos ovos durante seu armazenamento. Esses resultados são coerentes aos obtidos por Barbosa et al. (2008), Garcia et al. (2010), Figueiredo et al. (2011), Pissinati et al. (2014) e Giampietro-Ganeco et al. (2015). Foi observado a partir do $6^{\circ}$ dia de armazenamento que os ovos estocados em condições de temperatura ambiente apresentaram acentuadamente queda dos valores de Unidade Haugh, resultando em ovos de qualidade inferior.

Os valores de Unidade Haugh reduziram $(\mathrm{P}<0,05)$ linearmente à medida que aumentou o período de armazenamento dos ovos mantidos em temperatura ambiente e sob refrigeração, conforme as respectivas equações: $\hat{\mathrm{Y}}=85,366-$ $1,81364 x \quad\left(\mathrm{R}^{2}=0,91\right)$ e $\hat{\mathrm{Y}}=91,196$ $0,5169 x\left(\mathrm{R}^{2}=0,75\right)$.

Os ovos avaliados no dia da postura apresentaram inicialmente valor de Unidade Haugh igual a 94,62 e, aos 30 dias de armazenamento em temperatura ambiente, passaram a apresentar valor igual a 30,82, ou seja, os ovos perderam sua qualidade rapidamente, saindo do padrão de excelente qualidade ((AA valores de UH superiores a 72), para ovos de qualidade baixa (valores de UH menores que 60), conforme preconizado pelo USDA (2000). Essa reposta pode ser atribuída à redução na altura do albúmen, devido sua liquefação, processo este que foi acelerado pelas altas temperaturas do ambiente durante o período experimental.

Por outro lado, os ovos armazenados em condições de refrigeração apresentaram qualidade semelhante entre os ovos avaliados no primeiro e no $30^{\circ}$ dia, cujos valores médios foram de 94,36 e 79,19, respectivamente, permanecendo no padrão de qualidade excelente. Tal fato corroborou os resultados relatados por Jones \& Musgrove (2005), Barbosa et al. (2008), Xavier et al. (2008), Garcia et al. (2010) e Figueiredo et al. (2011) e GiampietroGaneco et al. (2015), demonstrando que o armazenamento do ovo sob refrigeração é essencialmente benéfico para a qualidade interna.

Os valores de diâmetro de albúmen, índice de albúmen e de gema dos ovos foram influenciados $(\mathrm{P}<0,05)$ pelas diferentes temperaturas e períodos de armazenamento (Tabela 3 ).

Os ovos mantidos armazenados à $26,5^{\circ} \mathrm{C}$ apresentaram maiores $(\mathrm{P}<0,05)$ diâmetros de albúmen que os ovos estocados sob refrigeração a partir do sexto dia de armazenamento, corroborando que a temperatura e o tempo de armazenamento interferem no tamanho do diâmetro de albúmen.

Os diâmetros de albúmen dos ovos aumentaram $(\mathrm{P}<0,05) \quad$ linearmente à medida que se prolongou o tempo de armazenamento, tanto para as condições de temperatura ambiente quanto para refrigeração, conforme as seguintes equações: $\quad \hat{Y}=\quad 78,7776+1,9234 x$ $\left(\mathrm{R}^{2}=0,95\right)$ e $\hat{\mathrm{Y}}=74,0132+0,1896 x \quad\left(\mathrm{R}^{2}=\right.$ $0,90)$, respectivamente. Esses resultados foram decorrentes da elevada temperatura e tempo de estocagem dos ovos, favorecendo o aumento do $\mathrm{pH}$ e do diâmetro e diminuição da altura do 
albúmen, com consequente queda na qualidade interna dos ovos.

Independente do período de armazenamento verificou-se os valores de índice de albúmen foram menores $(\mathrm{P}<0,05)$ quando os ovos foram estocados a temperatura ambiente comparados com os ovos mantidos sob refrigeração. $\mathrm{O}$ efeito da temperatura foi observado a partir do $6^{\circ}$ dia de armazenamento dos ovos em ambas temperaturas, apesar de ter sido constatado uma redução acentuada nos índices dos ovos mantidos a $26,5^{\circ} \mathrm{C}$, demonstrando que a refrigeração proporciona melhores índices de albúmen e consequentemente ovos de melhor qualidade.

Tabela 3. Valores de diâmetro de albúmen, índice de albúmen e de gema de ovos de poedeiras comerciais armazenados em diferentes temperatura e períodos de estocagem

\begin{tabular}{|c|c|c|c|c|c|c|}
\hline \multirow{2}{*}{$\begin{array}{l}\text { Período armazenamento } \\
\text { (dias)* }\end{array}$} & \multicolumn{2}{|c|}{$\begin{array}{l}\text { Diâmetro Albúmen } \\
(\mathrm{mm})\end{array}$} & \multicolumn{2}{|c|}{ Índice de Albúmen } & \multicolumn{2}{|c|}{ Índice de Gema } \\
\hline & $26,5^{\circ} \mathrm{C}$ & $7,3^{\circ} \mathrm{C}$ & $26,5^{\circ} \mathrm{C}$ & $7,3^{\circ} \mathrm{C}$ & $26,5^{\circ} \mathrm{C}$ & $7,3^{\circ} \mathrm{C}$ \\
\hline 0 & $73,83^{\mathrm{a}}$ & $73,83^{a}$ & $0,12^{\mathrm{a}}$ & $0,12^{\mathrm{a}}$ & $0,39^{a}$ & $0,39^{\mathrm{a}}$ \\
\hline 6 & $94,57^{\mathrm{a}}$ & $79,27^{\mathrm{b}}$ & $0,05^{\mathrm{b}}$ & $0,09^{\mathrm{a}}$ & $0,33^{b}$ & $0,39^{\mathrm{a}}$ \\
\hline 12 & $117,13^{\mathrm{a}}$ & $81,08^{\mathrm{b}}$ & $0,02^{b}$ & $0,08^{\mathrm{a}}$ & $0,26^{\mathrm{b}}$ & $0,40^{\mathrm{a}}$ \\
\hline 18 & $116,13^{a}$ & $79,22^{b}$ & $0,02^{\mathrm{b}}$ & $0,08^{\mathrm{a}}$ & $0,30^{\mathrm{b}}$ & $0,41^{\mathrm{a}}$ \\
\hline 24 & $134,36^{\mathrm{a}}$ & $79,74^{\mathrm{b}}$ & $0,02^{b}$ & $0,08^{\mathrm{a}}$ & $0,26^{\mathrm{b}}$ & $0,39^{\mathrm{a}}$ \\
\hline 30 & $130,48^{a}$ & $77,40^{\mathrm{b}}$ & $0,02^{\mathrm{b}}$ & $0,08^{\mathrm{a}}$ & $0,24^{\mathrm{b}}$ & $0,41^{\mathrm{a}}$ \\
\hline Médias & $111,08^{\mathrm{A}}$ & $78,42^{\mathrm{B}}$ & $0,04^{\mathrm{B}}$ & $0,09^{\mathrm{A}}$ & $0,30^{\mathrm{B}}$ & $0,40^{\mathrm{A}}$ \\
\hline CV (\%) & \multicolumn{2}{|c|}{10,57} & \multicolumn{2}{|c|}{20,19} & \multicolumn{2}{|c|}{6,12} \\
\hline
\end{tabular}

Médias seguidas de letras diferentes na coluna, para a mesma variável, diferem entre si, pelo teste Student Newman Keuls $(\mathrm{P}<0,05)$.

*Efeito linear $(\mathrm{P}<0,05)$.

O índice de albúmen dos ovos reduziu $(\mathrm{P}<0,05)$ de forma linear em função do prolongamento do período de armazenamento dos ovos submetidos tanto em temperatura ambiente quanto sob refrigeração, de acordo com as respectivas equações: $\hat{Y}=0,14511-$ $0,003895 x \quad\left(\mathrm{R}^{2}=0,93\right)$ e $\hat{\mathrm{Y}}=0,1258-$ $0,00676 x\left(R^{2}=0,95\right)$. Khan et al. (2013) relataram resultados semelhantes, pois uma vez que ocorre perda de $\mathrm{CO}_{2}$ do conteúdo interno dos ovos com o passar do período de estocagem, consequentemente, os valores de $\mathrm{pH}$ do albúmen se elevam, ocorrendo menores valores de unidade Haugh e alteração do sabor dos ovos, haja vista que o $\mathrm{pH}$ alcalino influencia negativamente a membrana vitelínica (LEANDRO et al., 2005). Scott \& Silversides (2000) encontraram respostas semelhantes ao armazenarem ovos por 10 dias à temperatura ambiente, pois os ovos apresentaram aumento no diâmetro de albúmen, diminuição na sua altura o que acarretou em menor índice de albúmen. Independente do período de armazenamento, os ovos mantidos em temperatura ambiente apresentaram menores $(\mathrm{P}<0,05)$ índices de gema quando comparados aos ovos mantidos em condições de refrigeração. Constatou-se o efeito da temperatura a partir do sexto dia para os ovos armazenados em condições de temperatura ambiente e sob 
refrigeração, embora a queda no índice de gema tenha sido mais evidente nos ovos mantidos em condições de temperatura ambiente.

Os índices de gema foram influenciados linearmente $(\mathrm{P}<0,05)$ à medida que se prolongou o tempo de armazenamento dos ovos em condições de temperatura ambiente e de refrigeração, segundo as equações: $\hat{Y}=0,3669-0,00423 x$ $\left(\mathrm{R}^{2}=0,99\right)$ e $\hat{\mathrm{Y}}=0,3984+0,00058 x$ $\left(\mathrm{R}^{2}=0,93\right)$. Esses resultados corroboram os encontrados por Siebel \& SouzaSoares (2004), Garcia et al. (2010) e Giampietro-Ganeco et al. (2015), porém discordam dos relatados por Souza \& Souza (1995) ao avaliarem a qualidade interna de ovos de codornas. Com a elevação da temperatura e tempo de armazenamento a água da albumina atravessa a membrana vitelínica por osmose e fica retida na gema. $\mathrm{O}$ excesso de agua na gema determina o aumento de seu volume, ocasionando o enfraquecimento da membrana vitelínica e tornando a gema maior e achatada (SIEBEL \& SOUZASOARES, 2004; PISSINATI et al., 2014). Pelo fato de o índice de gema ser baseado na relação entre a altura e o diâmetro da gema, o aumento da temperatura de conservação pode influenciar negativamente sobre essa variável conforme observado nesse experimento.

Pelo exposto, os ovos armazenados até seis dias após a postura se mantêm em padrão de alta qualidade em temperatura ambiente $\left(26,5^{\circ} \mathrm{C}\right)$; e quando armazenados em refrigeração $\left(7,3^{\circ} \mathrm{C}\right)$ mantêm padrão de excelente qualidade até os 30 dias, garantindo ao consumidor um produto saudável.

\section{REFERÊNCIAS}

BARBOSA, N.A.A.; SAKOMURA, N.K.; MENDONÇA, M.O.; FREITAS, E.R.; FERNANDES, J.B.K. Qualidade de ovos comerciais provenientes de poedeiras comerciais armazenados sob diferentes tempos e condições de ambientes. Ars Veterinária, v.24, n.2, p.127-133, 2008.

BRASIL. Ministério da Agricultura, Pecuária e Abastecimento. Regulamento de Inspeção Industrial e Sanitária de Produtos de Origem Animal. Decreto ${ }^{\circ}$ 30.691, de 29 de março de 1952, e alterações. Diário Oficial da União. Brasília, 1997. Disponível em: $<$ www.agricultura.gov.br $>$. Acesso em: 27 dez. 2016.

CARVALHO, F.B.; STRINGHINI, J.H.; JARDIM FILHO, R.M.; LEANDRO, N.S.M.; CAFE, M.B.; DEUS, H.A.S.B. Qualidade interna e da casca para ovos de poedeiras comerciais de diferentes linhagens e idades.

Ciencia Animal Brasileira, v.8, n.1, p.25-29, 2007.

ESTRADA, M.M.; GALEANO, L.F.; HERRERA, M.R.; RESTREPO, L.F. Efecto de la temperatura y el volteo durante el almacenamiento sobre la calidad del huevo comercial. Revista Colombiana de Ciencias Pecuárias, v.23, p.183-190, 2012.

FIGUEIREDO, T.C., CANÇADO, S.V., VIEGAS, R.P., REGGO, I.O.P., LARA, L.J.C., Souza, M.R., BAIÃO, N.C. Qualidade de ovos comerciais submetidos a diferentes condições de armazenamento. Arquivo Brasileiro de Medicina Veterinária e Zootecnia, v.63, n.3, p.712-720, 2011. 
FREITAS, L.W., PAZ, I.C.L.A., GARCIA, R.G., CALDARA, F.R., SENO, L.O., FELIX, G.A., LIMA, N.D.S., FERREIRA, V.M.O.S., CAVICHIOLO, F. Aspectos qualitativos de ovos comerciais submetidos a diferentes condições de armazenamento. Revista Agrarian, v.4, n.11, p.66-72, 2011.

GARCIA, E.R.M.; ORLANDI, C.C.B.; OLIVEIRA, C.A.L.; CRUZ, F.K.; SANTOS, T.M. B.; OTUTUMI, L.K. Qualidade de ovos de poedeiras semipesadas armazenados em diferentes temperaturas e períodos de estocagem.

Revista Brasileira de Saúde e Produção Animal [online], v.11, n.2, p.505-518, 2010.

GIAMPIETRO-GANECO, A.; BORBA, H.; SCATOLINI-SILVA, A.M.; BOIAGO, M.M.; SOUZA, P.A.; MELLO, J.L.M. Quality assessment of eggs packed under modified atmosphere. Ciência e Agrotecnologia, v.39, n.1, p.82-88, 2015.

HAMILTON, R.G.M. Methods and factors that affect the measurement of egg shell quality. Poultry Science, v.61, n.10, p.2022-2039, 1982.

JONES, D.R.; MUSGROVE, M.T. Effects of extended storage on egg quality factors. Poultry Science, v.84, n11., p.1774-1777, 2005.

KEENER, K.M.; LACROSSE, J.D.; BABSON, JK. Chemical method for determination of carbon dioxide content in egg yolk and egg albumen. Poultry Science, v.80, n.7, p.983-987, 2001.

KHAN, M.J.A.; KHAN, S.H.; BUKHSH, A.; ABBASS, M.I. JAVED, $M$. Effect of different storage period on egg weight, internal egg quality and hatchability characteristics of Fayumi eggs. Italian Journal Animal Science, v.12, n.2, p.323-328, 2013.

LEANDRO, N.S.M.; DEUS, H.A.B.; STRINGHINI, J.H.; CAFÉ, M.B.; ANDRADE, M.A.; CARVALHO, F.B. Aspectos de qualidade interna e externa de ovos comercializados em diferentes estabelecimentos na região de Goiânia. Ciência Animal Brasileira, v.6, n.2, p.71-78, 2005.

LOPES, L.L.R.A.; SILVA, Y.L.; NUNES, R.V.; TAKAHASHI, S.E.; MORI, C. Influência do tempo e das condições de armazenamento na qualidade de ovos comerciais. Revista Científica Eletrônica de Medicina Veterinária, Garça, ano IX, n.18, jan.2012. Disponível em:

$<$ www.revista.inf.br/veterinaria 18/artgos/ar t11.pdf>. Acesso em: 03 ago. 2015.

OLIVEIRA, G.E.; FIGUEIREDO T.C; SOUZA, M.R.; OLIVEIRA, A.L.; CANÇADO, S.V.; GLORIA, M.B.A. Bioactive amines and quality of egg from dekalb hen under different storage conditions. Poultry Science, v.88, n.11, p.2428-2434, 2009.

PISSINATI, A.; OBA, A.; YAMASHITA, F.; SILVA, C.A.; PINHEIRO, J.W.; ROMAN, J.M.M. Internal quality of eggs subjected to different types of coating and stored for 35 days at $25^{\circ} \mathrm{C}$. Semina: Ciências Agrárias, v.35, n.1, p.531-540, 2014.

RAMOS, K.C.B.T.; CAMARGO, A.M.; OLIVEIRA, E.C.D.; CEDRO, T.M.M.; MORENZ, M.J.F. Avaliação da idade da poedeira, da temperatura de armazenamento e do tipo embalagem sobre a qualidade de ovos comerciais.

Revista Séries Ciências da Vida, v.30, n.2, p.37-46, 2010. 
ROMANOFF, A.L.; ROMANOFF, A.J. The avian egg. 2.ed. New York: John Wiley \& Sons, 1963. 918p.

SAMLI, H.E.; AGMA, A.;

SENKOYLU, N. Effects of Storage

Time and Temperature on Egg Quality in Old Laying Hens. Journal of

Applied Poultry Research, v.14, n.3, p.548-553, 2005.

SANTOS, M.S.V.; ESPÍNDOLA, G.B.; LÔBO, R.N.B.; FREITAS, E.R.; GUERRA, J.L.L.; SANTOS, A.B.E. Efeito da temperatura e estocagem em ovos. Ciência e Tecnologia de

Alimentos, v.29, n.3, p.513-517. 2009.

SCOTT, T.A.; SILVERSIDES, T. B. The effect of storage and strain of hen on egg quality. Poultry Science, v.79, p.1725-1729, 2000.

SIEBEL, N.F.; SOUZA-SOARES, L.A. Efeito do resíduo de pescado sobre as características físicas e químicas de ovos de codornas armazenados em diferentes períodos. Semina: Ciências Agrárias, v.25, n.1, p.35-44, 2004.

SILVERSIDES, F.G., BUDGELL, K. The relationships among measures of egg albumen height, $\mathrm{pH}$, and whipping volume. Poultry Science, v.83, n.10, p.1619-1623, 2004.

SILVERSIDES, F.G.; SCOTT, T.A. Effect of storage and layer age on quality of eggs from two lines of hens. Poultry Science, v.80, n.8, p.12401245, 2001.

SILVERSIDES, F.G.; TWIZEYIMANA, F.; VILLENEUVE, P. A study relating to the validity of the Haugh unit correction for egg weight in fresh eggs. Poultry Science Journal, v.72, n.4, p.760-764, 1993.
SOUZA, H.B.; SOUZA, P. Efeito da temperatura de estocagem sobre a qualidade interna de ovos de codorna armazenados durante 21 dias.

Alimentos e Nutrição, v.6, n.1, p.7-13, 1995.

UNITED STATES DEPARTMENT OF AGRICULTURE - USDA. Egg-

Grading Manual. Washington: Departament of Agriculture. 2000. 56p. (Agricultural Markenting Service, 75).

UNIVERSIDADE FEDERAL DE VIÇOSA - UFV. Sistema de análises estatísticas e genéticas - SAEG.

Versão 9.1. Viçosa, MG, 2007. 59p.

XAVIER, I.M.C.; CANÇADO, S.V.; FIGUEIREDO, LARA, T.C.; LANA, A.M.Q; SOUZA, M.R.; BAIÃO, N.C. Qualidade de ovos de consumo submetidos a diferentes condições de armazenamento. Arquivo Brasileiro de Medicina Veterinária e Zootecnia, v.60, n.4, p.953-959, 2008.

Data de recebimento: 08/09/2015

Data de aprovação: 23/01/2017 